\title{
ESTANDARIZACIÓN Y OPTIMIZACIÓN DE PROCESOS DE VINO DE MORA DE CASTILLA (Rubus glucus Benth)
}

\author{
Autor: \\ Manuel Alberto Coronel Feijó'
}





\section{Resumen}

El objetivo principal fue encontrar la mejor combinación de sustrato y temperatura de fermentación para lograr un producto dentro de norma INEN con base al contenido alcohólico $\left(5-23^{\circ} \mathrm{GL}\right)$. El estudio se realizó en la Planta Piloto de Alimentos y Laboratorio de Biotecnología Alimentaria de la Universidad Tecnológica Equinoccial. Se estudió el proceso de elaboración de vino de mora de Castilla a partir de mosto acondicionado con tres niveles de concentración de sustrato fermentable (15, 20 y 25 grados Brix) a temperatura variable (15, 20 y 25 grados centígrados) en un Bioreactor semi automático de 60 litros de capacidad. Se utilizó levadura (Saccharomyces cerevisiae) previamente adaptada en cada mosto a estudiar $20^{\circ} \mathrm{C}$ durante 1 hora. Se aplicó un diseño factorial $3^{2}$ para determinar la cinética de fermentación por cada tratamiento. Para los tres niveles de temperatura, la tasa máxima de agotamiento de sustrato se alcanzó alrededor del décimo día con mostos de $15^{\circ}$ Brix inicial. Finalmente se aplicó un análisis sensorial (sobre vino clarificado por una semana a $7{ }^{\circ} \mathrm{C}$ en cámara fría) con una prueba de aceptación simple por cada tratamiento y el mayor puntaje lo obtuvo la combinación $20^{\circ} \mathrm{C}-20^{\circ} \mathrm{Bx}$. Bajas temperaturas lograron vinos con mayor perfil organoléptico en tiempos más prolongados. Altas temperaturas logran vinos con menor perfil organoléptico en cortos tiempos.

Palabras clave: Mora de Castilla, mosto, grados Brix, Bioreactor, levadura, sustrato, cinética defermentación.

\section{Abstract}

The principal objective of this study was to find the best combination of substrate and fermentation temperature, to make a product according to the norms of the Ecuadorian Normalized Institute to the alcoholic level ( $5-23 \mathrm{GL})$. The study was done in the Food Plant and the Food Biotechnology Laboratory in Occidental campus of the Universidad Tecnológica Equinoccial. The blackberry wine process was studied based on three levels of substrate (15, 20 and 25 Brix) and three temperatures (15, 20 and 25 Celsius) in a semiautomatic Bioreactor for 60 liters of capacity. By using yeast (Saccharomyces cerevisiae) previously adapted at $21 \mathrm{C}$ during 1 hour. A factorial design 32 to determine kinetic of fermentation for each one. For three levels of temperature, the maximum rate to substrate decreasing was becoming in tenth day with $15^{\circ}$ Brix. Finally, an acceptability test (on wine, clarified for one week in $7^{\circ} \mathrm{C}$ in a cold room) was applied and the best score was the $20^{\circ} \mathrm{C}-20^{\circ} \mathrm{Bx}$ combinations. Low temperatures got wines with more organoleptic profile in more time. High temperatures got wines with less organoleptic profile in short time.

Keywords: Castilla blackberry, fruit juice, Brix degrees, Bioreactor, yeast, substrate, fermentation Kinetics. 


\section{Introducción}

La producción nacional de mora registra una expansión constante, lo que hace suponer que sus perspectivas son promisorias y que puede convertirse en una excelente alternativa para diversificar las exportaciones. La de mayor importancia en nuestro país es $R$. glaucus con sus principales variedades: Brazos y Mora de Castilla. En Ecuador se la cultiva en varias provincias siendo las principales Carchi, Pichincha, Tungurahua, Chimborazo y Bolívar (Villacrés, 1985). La mora de Castilla es una fruta con gran aceptación tanto para su consumo en fresco por su exquisito sabor, aroma y atractivo color, así como por la facilidad para su industrialización como materia prima para la preparación de dulces, mermeladas, jugos, helados, arropes y, eventualmente, vino(Ortiz, 2008).

Hay cuatro factores críticos que influyen en la calidad final de la mora: la manipulación, la temperatura, el tiempo transcurrido desde la cosecha y el tamaño del empaque. Dentro de los beneficios de esta fruta a la salud se pueden mencionar: buena para el corazón, para la circulación y problemas de la piel, excelente remedio contra la diarrea, poderosas propiedades antioxidantes por su combinación de vitaminas C y E (21 mg./ $100 \mathrm{~g}$ y $0.71 \mathrm{mg} . / 100 \mathrm{~g}$, respectivamente) y es rica en pectina, una fibra soluble que ayuda a reducir los niveles de colesterol en sangre (Cáceres, 2003).

Se ha descrito que las especies de Rubus tienen importantes compuestos fenólicos que pueden presentar dentro de la célula actividad antioxidante, reduciendo la concentración de radicales libres y, en algunos casos, logran establecer grupos de quelación con iones metálicos, pudiendo reducir trombosis, activar macrófagos e inhibir la peroxidación (D. García, Viloria-Matos, B., \& Moreno-Álvarez, 2003). Los flavonoides se encuentran en todas las plantas, pero están localizados principalmente en las vacuolas de las células de la piel de frutas y hortalizas, aportando parte del sabor y del color; la mayoría son solubles en agua y no son sintetizados por el cuerpo humano ni producidos sintéticamente. Los usos de los flavonoides dependen de su forma de combinarse; cada planta contiene una única combinación de flavonoides (Ochoa \& Ayala, 2004). Una dieta rica en frutas y vegetales está asociada con reducción en el riesgo de padecer cáncer, enfermedades cardiovasculares, y trastornos neurodegenerativos asociados con la edad (Vasco, 2009).

En otras latitudes se ha investigado la capacidad antioxidante de vinos de frutas tropicales, particularmente con la guayaba, piña, naranja, papaya y guanábana. En general, los vinos de frutas tropicales exhibieron una actividad antioxidante similar a los vinos blancos de uva. Los vinos de naranja y de guayaba se destacaron por poseer la mayor actividad antirradical y poder reductor que el resto de los vinos estudiados (Rodríguez, Valdés, \& Queris, 2007).

Al trabajar con vinos de frutas nos enfrentamos al reto tecnológico de hacer transformaciones a nuestra materia prima para lograr un producto de óptima calidad. No se dispone en este sentido del conocimiento como se tiene sobre la uva, en el que todas las condiciones están dadas para obtener vino. Estas transformaciones están dirigidas a asegurar tres aspectos fundamentales: viabilidad del proceso, optimización organoléptica y la rentabilidad (M. García, Quintero, \& López-Munguía, 2000).

El producto a elaborar (vino de frutas) será el resultante de un proceso bioquímico, llamado fermentación alcohólica, que requiere condiciones muy específicas para culminar con éxito. Tendrá que poseer las características de sabor, aroma, color, untuosidad, etc, que sean agradables al consumidor. Para lograr esto, el técnico deberá considerar las características intrínsecas de la fruta a emplear y, de ser necesario, podrá diluir, hacer mezclas, agregar aditivos o reforzar sabores. La acidez y el contenido de azúcar entran también en juego en el aspecto organoléptico pero ahora como elementos delineantes del sabor (Grainger \& Tattersall, 2005).

La rentabilidad de una fruta para elaborar vino será el producto de una combinación de diversos factores que el técnico 
deberá saber administrar. Está, por ejemplo, el rendimiento en jugo o pulpa, sus características de acidez y dulzor, la riqueza de elementos aromáticos, los costos de la fruta y su procesamiento, su disponibilidad geográfica, la estacionalidad, etc. El contenido alcohólico procede casi en su totalidad de la fermentación del jugo de fruta (Hoyos \& Vélez, 2000).

Los vinos de fruta en Ecuador están. prácticamente, circunscritos a fábricas artesanales y su producción industrial es casi inexistente. Se conoce, de la elaboración a escala comercial en la provincia de Zamora la marca Rancho Alegre para productos a base de papaya o maracuyá y, si bien los volúmenes de producción no son muy altos, ha podido conservar una posición al operar desde 1950. Las investigaciones al respecto son escasas. Se hizo un estudio comparativo entre levadura panadera y vínica, su relación con la turbidez y el grado alcohólico logrado del vino de mora. Por otro lado, se demostró que entre la maceración con agua fría y caliente no había diferencia significativa (Villacrés, 1985). Estas experiencias permitieron partir de dos hechos, utilizar levadura panadera y macerar en agua fría los restos vegetales de la mora en conjunto con el mosto en fermentación.

Con base en lo anterior, se propone la elaboración del vino a partir de mora de Castilla por la elevada producción nacional de esta fruta y sus potenciales características organolépticas. En adelante se ajustarán los parámetros de proceso para incrementar el perfil organoléptico del producto final. Además, en concordancia con la tecnología del vino de uva, se aplican técnicas que no destruyen los tejidos vegetales, liberando así, sabores y aromas potencialmente extraños (INEN 247, 2010).

Con la estandarización y optimización en la elaboración del Vino de Mora de Castilla se podrá contribuir hacia un producto comercialmente aceptable.

\section{Materiales y Métodos}

Se utilizó mora $\left(10^{\circ} \mathrm{Bx}\right.$. y $\mathrm{pH}=3.3$ e IM = 5.5) proveniente de la provincia de Tungurahua, cantón Patate. El índice de madurez se tomó de la norma INEN para frutas frescas de mora de castilla (INEN 247, 2010).

Se acondicionó mosto de mora (extraído por prensado) con agua purificada (relación 1:1) comercial y azúcar refinada hasta completar los grados Brix deseados para tres niveles a estudiar (15, 20 y $25^{\circ} \mathrm{Bx}$.). Se reguló el $\mathrm{pH}$ a un valor de 3,2. Como aditivo permitido se utilizó 200 ppm de meta bisulfito de sodio para el mosto antes de su fermentación.

Para el estudio de la cinética de fermentación, las unidades experimentales eran de $2 \mathrm{~kg}$ demosto acondicionado, en contenedores pequeños y utilizando como fuente de calefacción un Baño de María marca Hannah con control automático de temperatura. Para cada temperatura $\left(15,20\right.$ y $\left.25^{\circ} \mathrm{C}\right)$ se introdujeron tres contenedores con tres niveles de Brix. Las gráficas se obtuvieron enfrentando Brix versus tiempo de fermentación.

Para la lectura de los grados Brix se utilizó un refractómetro Hannah escala 0 - 32. Los grados alcohólicos se dedujeron a partir de la escala Brix residual con base a la ecuación de regresión lineal de la tabla de Tecnología de Vinos de Frutas (Arozarena, 2007). Con estos datos se obtenía la masa volúmica (densidad) que tiene su equivalente en grados Gay - Lussac $\left({ }^{\circ} \mathrm{GL}\right)$

Así:

$y=a+b x$

Donde:

\begin{tabular}{|c|c|c|}
\hline $\mathrm{Y}$ & \multicolumn{2}{|c|}{ masa volúmica } \\
\hline $\mathrm{a}$ & $\mathbf{- 1 . 9 9 1 8}$ & $\mathrm{k}$ \\
\hline $\mathrm{b}$ & $\mathbf{0 . 6 7 3 2 \mathrm { k }}$ & \\
\hline $\mathrm{X}$ & grados & Brix \\
\hline
\end{tabular}


Las tablas de regresión pueden aplicarse con otros datos experimentales como grados Baumé o densidad (masa volúmica).

La fermentación a escala piloto se realizó en Bioreactor diseñado en la Universidad Tecnológica Equinoccial. Está fabricado en acero inoxidable $\mathrm{AISI} 304$, con 60 litros de capacidad, posee controles de temperatura, presión y agitación, visores y válvulas sanitarias para la toma de muestras.

El tratamiento $\mathrm{T} 2 \mathrm{~B} 2\left(20^{\circ} \mathrm{C}, 20 \mathrm{Bx}\right.$.) se llevó al Bioreactor de escala piloto de 60 litros de capacidad.

\section{Resultados y Discusión}

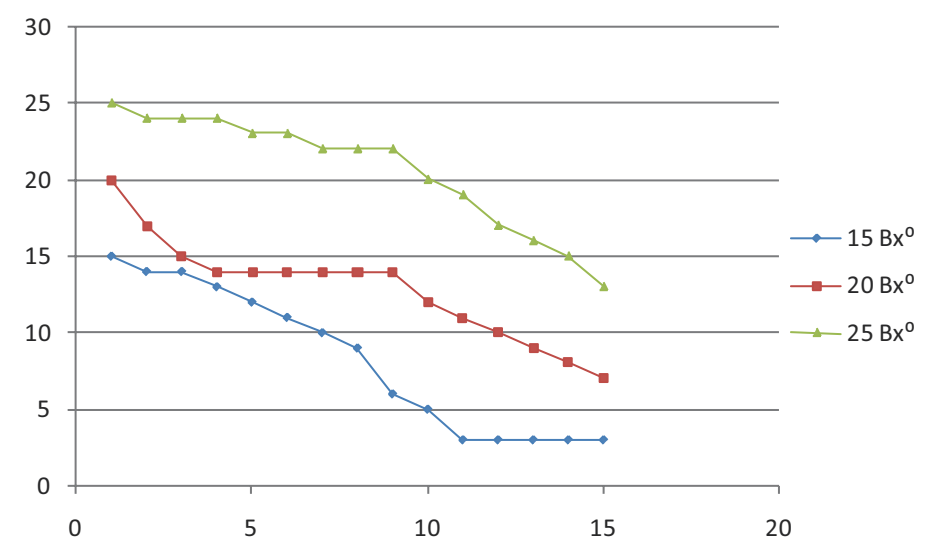

Figura 1. Agotamiento de sustrato ( ${ }^{0}$ Brix) en función del tiempo

(días) a $15^{\circ} \mathrm{C}$ para tres concentraciones iniciales de mosto.

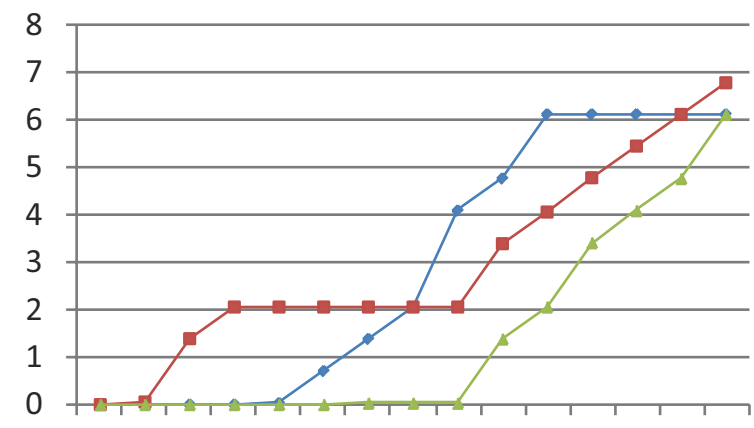

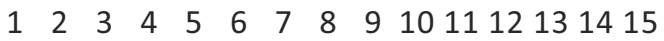

Figura 2. Formación de alcohol ( ${ }^{\circ} \mathrm{GL}$ ) en función del tiempo (días) a $15^{\circ} \mathrm{C}$.
En la figura 1 se aprecia que para la curva de $15^{\circ} \mathrm{Bx}$., se estabiliza el contenido residual de azúcares en $3{ }^{\circ} \mathrm{Bx}$. a los 11 días de fermentación; en las curvas para 20 y $25^{\circ} \mathrm{Bx}$, se estabiliza a los 15 días con un contenido residual de azúcares de 7 y $13^{\circ} \mathrm{Bx}$. respectivamente. En la figura 2 se aprecia el incremento de contenido alcohólico máximo para la curva de $20^{\circ} \mathrm{Bx} .\left(6.8^{\circ} \mathrm{GL}\right)$ a los 15 días, en relación a las curvas de 15 y $25^{\circ} \mathrm{Bx}$., con contenidos de $6.1^{\circ} \mathrm{GL}$ en ambos casos.

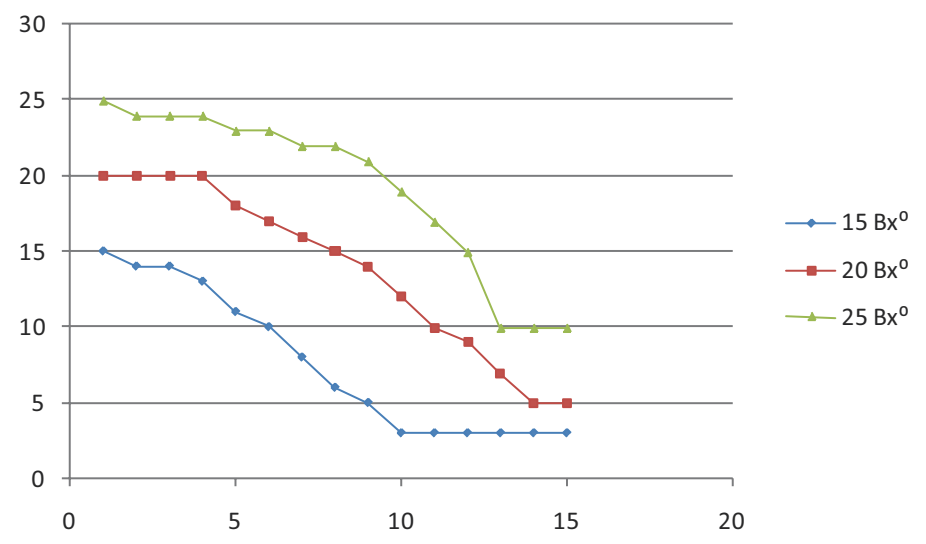

Figura 3.Agotamiento de sustrato ( ${ }^{\circ}$ Brix) en función del tiempo (días) a $20^{\circ} \mathrm{C}$ para tres concentraciones iniciales de mosto.

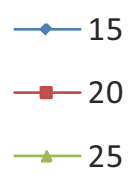


En la figura 3 se observa que la curva de $15^{\circ} \mathrm{Bx}$. se estabiliza a los 10 días de fermentación con un contenido residual de azúcares en $3{ }^{\circ} \mathrm{Bx}$. En la curva para $20{ }^{\circ} \mathrm{Bx}$. lo hace a los 14 días (residual de 4 ${ }^{\circ} \mathrm{Bx}$.) y en la de $25^{\circ} \mathrm{Bx}$. a los 13 días ( $10^{\circ} \mathrm{Bx}$. de residual). En la figura 4 se aprecia un incremento en contenido alcohólico máximo para las curvas de 20 y $25^{\circ} \mathrm{Bx}$. a los 15 días de fermentación con un valor de 8.1 ${ }^{\circ} \mathrm{GL}$ en ambos casos. La curva de $15^{\circ} \mathrm{Bx}$. alcanza su máximo contenido a los 10 días (6.1 GL).

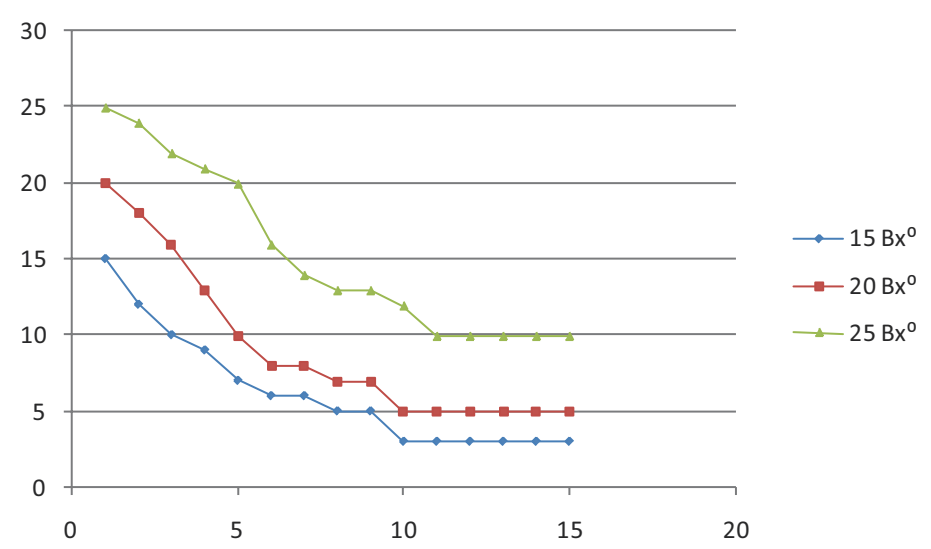

Figura 5. Agotamiento de sustrato ( ${ }^{\circ}$ Brix) en función del tiempo (días) a $25^{\circ} \mathrm{C}$ para tres concentraciones iniciales de mosto.

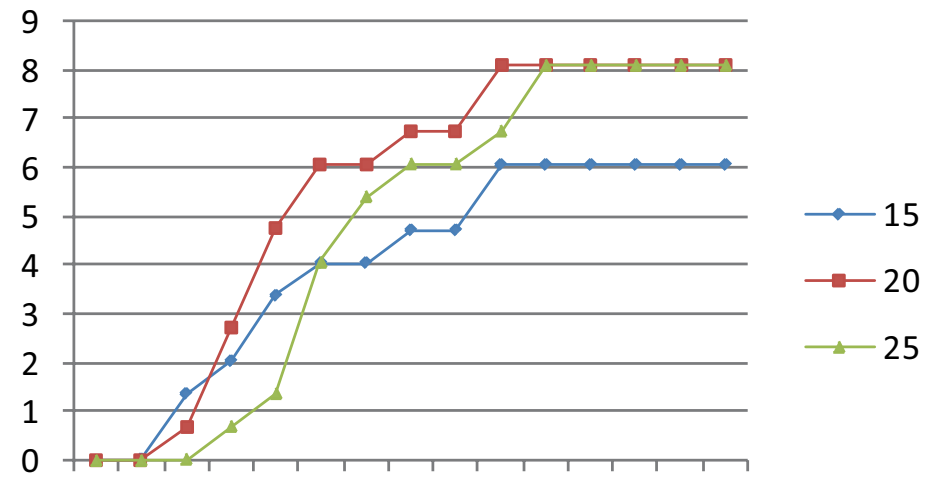

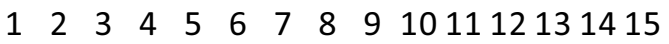

Figura 6. Formación de alcohol ( ${ }^{\circ} \mathrm{GL}$ ) en función del tiempo (días) a $25^{\circ} \mathrm{C}$.
En la figura 5 se observa que la curva de $15^{\circ} \mathrm{Bx}$. se estabiliza a los 10 días ( $3^{\circ} \mathrm{Bx}$. residuales). Para las curvas de 20 y $25^{\circ} \mathrm{Bx}$. se estabilizan a los 10 y 11 días con residual de 5 y $10^{\circ} \mathrm{Bx}$. respectivamente. En la figura 6, el contenido alcohólico máximo para la curva de $15^{\circ} \mathrm{Bx}$. es de 6.1 GL. Para la curva $20^{\circ} \mathrm{Bx}$. ocurre al día 10 (8.1 GL), mientras que en la curva de $25^{\circ} \mathrm{Bx}$. ocurre al día número $11\left(8.1^{\circ} \mathrm{GL}\right)$.

Tabla 1. Informe Técnico del Vino de Mora (T2B2) con 30 días de reposo

\begin{tabular}{|c|c|c|c|c|c|}
\hline \multicolumn{6}{|c|}{ Especificaciones INEN 37} \\
\hline EnsayosFísico - Químicos & Método & Unidad & Resultado & $m$ & $M$ \\
\hline Acidezvolátil & INEN 341 & $\%$ & 0.12 & $\ldots$ & 1.5 \\
\hline Acidez total & INEN 341 & $\%$ & 10.36 & 4 & 14 \\
\hline Metanol & INEN 347 & $\%$ & 0.029 & $\ldots$ & 0.5 \\
\hline Gradoalcohólico & INEN 340 & ${ }^{\circ} \mathrm{GL}$ & 12 & 8 & 18 \\
\hline Ceniza & INEN 348 & $\%$ & 1.47 & 1.4 & $\ldots$ \\
\hline Cloruros & Mohr & $\mathrm{g} / \mathrm{l}$ & 0.2 & $\ldots$ & 1 \\
\hline Anhídrido sulfuroso libre & INEN 357 & $\mathrm{gSO} 2 / \mathrm{l}$ & 0.041 & $\ldots$ & 0.35 \\
\hline Ensayosorganolépticos & Método & Unidad & Resultado & & \\
\hline Color & sensorial & $\ldots$ & rojo oscuro & & \\
\hline Olor & sensorial & $\ldots$ & característico & & \\
\hline Sabor & sensorial & $\ldots$ & característico & & \\
\hline
\end{tabular}

Laboratorios SEIDLA (Servicio Integral de Laboratorio)

En la tabla 1 de resultados (Informe Técnico de Laboratorio Acreditado) se aprecia que todos los parámetros se encuentran dentro de norma. Especial importancia reviste la acidez total. En vinos jóvenes, ésta sería la alteración más grave que incrementa, en mayor medida, la concentración de ácido acético, y que puede conducir al avinagramiento total del vino (Arozarena, 2007).

En la tabla 2, los valores numéricos nos indican el grado de aceptabilidad de un grupo de 25 personas. Un $64 \%$ son varones y el restante $36 \%$ mujeres. Del grupo encuestado, el $56 \%$ consume regularmente vino y de este subgrupo, el $80 \%$ consume vinos de frutas. 
Tabla 2. Aceptabilidad del vino de mora de Castilla

\begin{tabular}{|c|c|c|c|c|c|}
\hline \multicolumn{6}{|c|}{ Participantes del M unicipio de Pelileo } \\
\hline \multicolumn{6}{|c|}{ Características de la Población: } \\
\hline Número de consumidores encuestados: & & 25 & & & \\
\hline Hombres: & & 16 & $64 \%$ & & \\
\hline M ujeres: & & 9 & $36 \%$ & & \\
\hline \multicolumn{6}{|l|}{ Edad: } \\
\hline \multirow[t]{3}{*}{ Actividad L aboral: } & & & A grícola & 19 & $76 \%$ \\
\hline & & & Profesional & 3 & $12 \%$ \\
\hline & & & no contesta & 3 & $12 \%$ \\
\hline \multirow[t]{3}{*}{ Consume regularmente: } & $\mathrm{Si}$ & 14 & $56 \%$ & & \\
\hline & No & 9 & $36 \%$ & & \\
\hline & no responde & 2 & $8 \%$ & & \\
\hline \multirow[t]{3}{*}{ Ha consumido vino de frutas: } & $\mathrm{Si}$ & 20 & $80 \%$ & & \\
\hline & No & 4 & $16 \%$ & & \\
\hline & no responde & 1 & $4 \%$ & & \\
\hline & & & & & \\
\hline M e gusta mucho & & 15 & $60 \%$ & & \\
\hline M e gusta un poco & & 10 & $40 \%$ & & \\
\hline No me gusta ni me disgusta: & & 0 & $0 \%$ & & \\
\hline Me disgusta un poco: & & 0 & $0 \%$ & & \\
\hline me disguta mucho: & & 0 & $0 \%$ & & \\
\hline \multirow[t]{3}{*}{ ¿Compraría este producto? } & $\mathrm{Si}$ & 22 & $88 \%$ & & \\
\hline & No & 1 & $4 \%$ & & \\
\hline & no responde & 2 & $8 \%$ & & \\
\hline
\end{tabular}

- En relación a la materia prima, el tratamiento de prensado, se hizo manualmente. Este hecho, permitió liberar el contenido sin destruir el centro de la fruta, rico en pectinas y que contribuye a su vez a la turbidez del producto fermentado. Caso similar se practica con las uvas aunque con sistemas altamente mecanizados.

- La levadura panadera (Saccharomyces cerevisiae), y la maceración en frío paralelamente a la fermentación, según recomienda Villacrés (1985) permitieron agotar el sustrato hasta niveles de 3 grados Brix en promedio.

- La Sulfitación recomendada para vinos de frutas por Arozarena (2007), permitió mantener un adecuado nivel de anaerobiosis e higiene en el mosto en fermentación. Los análisis efectuados de sulfito libre corroboran las exigencias de las normas INEN para esta clase de productos.

\section{Conclusiones}

A partir de los resultados obtenidos se observa que en las condiciones de $20^{\circ} \mathrm{Bx}$. para el mosto de inicio y $20^{\circ} \mathrm{C}$ de temperatura de fermentación se alcanza el máximo contenido de alcohol $\left(8.1^{\circ} \mathrm{GL}\right)$ acorde con la norma INEN de vinos de fruta $\left(5-23^{\circ} \mathrm{GL}\right)$.

La levadura utilizada (Saccharomyces cerevisiae) tiene un límite de capacidad fermentativa evidenciada en los grados Brix residuales para las combinaciones propuestas (temperatura - Brix de inicio).

Los análisis de laboratorio muestran que los indicadores se encuentran dentro de los rangos de la norma INEN para vinos de frutas.

Después de la etapa en el bioreactor, el producto continúa una muy lenta fermentación en reposo / clarificación. Este valor dependerá del tiempo y de las condiciones de almacenamiento.

\section{Recomendaciones}

Realizar estudios de la clarificación natural y por medio de aditivos en relación a los componentes del vino. Estos posteriores estudios deberían realizarse con combinaciones de enzimas tipo pectinasas en el mosto de inicio.

Extender el estudio a la producción de vinagre y bebidas destiladas.

Estudiar el proceso de microfiltración tangencial para vino con y sin reposo, como ejemplo tecnología emergente.

En relación al proyecto de reglamento técnico ecuatoriano para bebidas alcohólicas, se requiere oficializar la normativa respecto a vinos de frutas. 


\section{Bibliografía}

Arozarena, I. (2007). Seminarios Internacionales. Paper presented at the Elaboración de Vinos de Frutas, Ambato, Ecuador.

Cáceres, H. (2003). Estudio preliminar del efecto de la microfiltración tangencial sobre la capacidad antioxidante en jugos de fruta clarificados., Escuela Agrícola Panamericana.

García, D., Viloria-Matos, A., B., D., \& Moreno-Álvarez, M. (2003). Características físico-químicas y composición de ácidos grasos del aceite crudo extraído de residuos de mora (Rubus glaucus Benth). Grasas y Aceites, 54(3), 259-263.

García, M., Quintero, R., \& López-Munguía, A. (2000). Biotecnología Alimentaria (Grupo Noriega Editores ed.). México D.F.: Editorial Limusa S.A.

Grainger, K., \& Tattersall, H. (2005). Producción de Vino: Desde la vid hasta la Botella (E. A. S.A., Trans. Blackwell Publishing Ltd. ed.). Zaragoza (España): Editorial Acribia S.A.

Hoyos, O., \& Vélez, P. (2000). Análisis de Alimentos: Manual de prácticas de laboratorio. Popayán: Universidad del Cauca.

INEN 247. (2010). Norma Técnica Ecuatoriana. Frutas Frescas. Mora (Primera ed., Vol. NTE INEN 2 247:2010): Instituto Ecuatoriano de Normalización.

Ochoa, C., \& Ayala, A. (2004). Los Flavonoides: Apuntes Generales y su Aplicación en la Industria de Alimentos. Ingeniería y competitividad, 6(2), 96-104.

Ortiz, J. (2008). Utilización de preparados Enzimáticos en la Producción de Vino de Mora, (Rubus glaucus Benth) 1-9.

Rodríguez, J., Valdés, O., \& Queris, O. (2007). Actividad Antioxidante de Vinos Elaborados con Frutas Tropicales. Ciencia y Tecnología de Alimentos, 17(2), 66-68.
Vasco, C. (2009). Phenolic Compounds in Ecuadorian Fruits.Unpublished Doctoral Thesis, University of Agricultural Sciences (SLU), Upppsala.

Villacrés, E. (1985). Elaboración de Vino de Mora. Universidad Técnica de Ambato. 
Anexos

TASA DE AGOTAMIENTO DE SUSTRATO EN QUINCE DÍAS DE FERMENTACIÓN

Día 1

\begin{tabular}{|l|c|c|c|}
\hline $\mathbf{B x}^{\mathbf{0}} / \mathbf{T}^{\mathbf{0}}$ & $\mathbf{1 5}{ }^{\circ} \mathbf{C}$ & $\mathbf{2 0}{ }^{\circ} \mathbf{C}$ & $25^{\circ} \mathbf{C}$ \\
\hline $\mathbf{1 5} \mathbf{B x}^{\mathbf{0}}$ & 15 & 15 & 15 \\
\hline $\mathbf{2 0} \mathbf{B x}^{\mathbf{0}}$ & 20 & 20 & 20 \\
\hline $25 \mathbf{B x}^{\mathbf{0}}$ & 25 & 25 & 25 \\
\hline
\end{tabular}

Día 2

\begin{tabular}{|l|c|c|c|}
\hline $\mathbf{B x}^{0} / \mathbf{T}^{\mathbf{0}}$ & $15^{\circ} \mathrm{C}$ & $\mathbf{2 0}{ }^{\circ} \mathrm{C}$ & $25^{\circ} \mathrm{C}$ \\
\hline $15 \mathbf{B x}^{\mathbf{0}}$ & 14 & 14 & 12 \\
\hline $20 \mathbf{B x}^{\mathbf{0}}$ & 17 & 20 & 18 \\
\hline $25 \mathbf{B x}^{0}$ & 24 & 24 & 24 \\
\hline
\end{tabular}

Día 3

\begin{tabular}{|l|c|c|c|}
\hline $\mathbf{B x}^{\circ} / \mathbf{T}^{\mathbf{0}}$ & $15{ }^{\circ} \mathbf{C}$ & $20^{\circ} \mathbf{C}$ & $25^{\circ} \mathbf{C}$ \\
\hline $15 \mathbf{B x}^{\mathbf{0}}$ & 14 & 14 & 10 \\
\hline $20 \mathbf{B x}^{\mathbf{0}}$ & 15 & 20 & 16 \\
\hline $25 \mathbf{B x}^{\mathbf{0}}$ & 24 & 24 & 22 \\
\hline
\end{tabular}

Día 4

\begin{tabular}{|l|c|c|c|}
\hline $\mathbf{B x}^{\circ} / \mathbf{T}^{\mathbf{0}}$ & $15^{\circ} \mathrm{C}$ & $\mathbf{2 0}{ }^{\circ} \mathbf{C}$ & $\mathbf{2 5}^{\circ} \mathbf{C}$ \\
\hline $15 \mathbf{B x}^{\mathbf{0}}$ & 13 & 13 & 9 \\
\hline $20 \mathbf{B x}^{\mathbf{0}}$ & 14 & 20 & 13 \\
\hline $25 \mathbf{B x}^{\mathbf{0}}$ & 24 & 24 & 24 \\
\hline
\end{tabular}

\begin{tabular}{|l|c|c|c|}
\hline \multicolumn{4}{|c|}{ Día } \\
\hline $\mathrm{Bx}^{\mathbf{0}} / \mathbf{T}^{\mathbf{0}}$ & $\mathbf{1 5}{ }^{\circ} \mathrm{C}$ & $\mathbf{2 0}{ }^{\circ} \mathrm{C}$ & $\mathbf{2 5}{ }^{\circ} \mathrm{C}$ \\
\hline $\mathbf{1 5} \mathbf{B x}^{\mathbf{0}}$ & 12 & 11 & 7 \\
\hline $\mathbf{2 0} \mathbf{B x}^{\mathbf{0}}$ & 14 & 18 & 10 \\
\hline $\mathbf{2 5} \mathbf{B x}^{\mathbf{0}}$ & 23 & 23 & 20 \\
\hline
\end{tabular}

\begin{tabular}{|l|c|c|c|}
\hline \multicolumn{5}{|c|}{ Día 6} \\
\hline $\mathbf{B x}^{\mathbf{0}} / \mathbf{T}^{\mathbf{0}}$ & $15^{\circ} \mathrm{C}$ & $\mathbf{2 0}{ }^{\circ} \mathrm{C}$ & $\mathbf{2 5}{ }^{\circ} \mathrm{C}$ \\
\hline $\mathbf{1 5} \mathbf{B x}^{\mathbf{0}}$ & 11 & 10 & 6 \\
\hline $\mathbf{2 0} \mathbf{B x}^{\mathbf{0}}$ & 14 & 17 & 8 \\
\hline $\mathbf{2 5} \mathbf{B x}^{\mathbf{0}}$ & 20 & 23 & 16 \\
\hline
\end{tabular}

\begin{tabular}{|c|c|c|c|}
\hline \multicolumn{4}{|c|}{ Día 7} \\
\hline $\mathrm{Bx}^{0} / \mathrm{T}^{\mathrm{o}}$ & $15^{\circ} \mathrm{C}$ & $20^{\circ} \mathrm{C}$ & $25^{\circ} \mathrm{C}$ \\
\hline $15 \mathrm{Bx}^{0}$ & 10 & 8 & 6 \\
\hline $20 \mathrm{Bx}^{\circ}$ & 14 & 16 & 8 \\
\hline $25 \mathrm{Bx}^{\circ}$ & 23 & 22 & 12 \\
\hline
\end{tabular}

\begin{tabular}{|l|c|c|c|}
\hline \multicolumn{5}{|c|}{ Día 8} \\
\hline $\mathrm{Bx}^{\circ} / \mathrm{T}^{\mathbf{0}}$ & $15^{\circ} \mathrm{C}$ & $\mathbf{2 0}{ }^{\circ} \mathrm{C}$ & $\mathbf{2 5}{ }^{\circ} \mathrm{C}$ \\
\hline $15 \mathrm{Bx}^{\mathbf{0}}$ & 9 & 6 & 5 \\
\hline $20 \mathrm{Bx}^{\mathbf{0}}$ & 14 & 15 & 7 \\
\hline $25 \mathrm{Bx}^{\mathbf{0}}$ & 22 & 22 & 16 \\
\hline
\end{tabular}

\begin{tabular}{|l|c|c|c|}
\hline \multicolumn{5}{|c|}{ Día 9 } \\
\hline $\mathbf{B x} / \mathrm{T}^{\mathbf{0}}$ & $15^{\circ} \mathrm{C}$ & $\mathbf{2 0}{ }^{\circ} \mathrm{C}$ & $25^{\circ} \mathrm{C}$ \\
\hline $15 \mathrm{Bx}^{0}$ & 6 & 5 & 5 \\
\hline $20 \mathrm{Bx}^{0}$ & 14 & 14 & 7 \\
\hline $25 \mathrm{Bx}^{\circ}$ & 22 & 21 & 16 \\
\hline
\end{tabular}

\begin{tabular}{|c|c|c|c|}
\hline \multicolumn{4}{|c|}{ Día 10} \\
\hline $\mathrm{Bx}^{0} / \mathrm{T}^{\mathrm{O}}$ & $15^{\circ} \mathrm{C}$ & $20^{\circ} \mathrm{C}$ & $25^{\circ} \mathrm{C}$ \\
\hline $15 \mathrm{Bx}^{0}$ & 5 & 3 & 3 \\
\hline $20 \mathrm{Bx}^{\circ}$ & 12 & 12 & 5 \\
\hline $25 \mathrm{Bx}^{\circ}$ & 20 & 19 & 12 \\
\hline
\end{tabular}

\begin{tabular}{|c|c|c|c|}
\hline \multicolumn{4}{|c|}{ Día 11} \\
\hline $\mathrm{Bx}^{0} / \mathrm{T}^{\mathrm{o}}$ & $15^{\circ} \mathrm{C}$ & $20^{\circ} \mathrm{C}$ & $25^{\circ} \mathrm{C}$ \\
\hline $15 \mathrm{Bx}^{\circ}$ & 3 & 3 & 3 \\
\hline $20 \mathrm{Bx}^{\circ}$ & 11 & 10 & 5 \\
\hline $25 \mathrm{Bx}^{\circ}$ & 19 & 17 & 10 \\
\hline
\end{tabular}

\begin{tabular}{|l|c|c|c|}
\hline \multicolumn{5}{|c|}{ Día $\mathbf{1 2}$} \\
\hline $\mathbf{B x} \mathbf{T}^{\mathbf{0}}$ & $\mathbf{1 5}{ }^{\circ} \mathrm{C}$ & $\mathbf{2 0}{ }^{\circ} \mathrm{C}$ & $\mathbf{2 5}{ }^{\circ} \mathrm{C}$ \\
\hline $\mathbf{1 5} \mathbf{B x}^{\mathbf{0}}$ & 3 & 3 & 3 \\
\hline $20 \mathrm{Bx}^{\mathbf{0}}$ & 10 & 9 & 5 \\
\hline $\mathbf{2 5} \mathrm{Bx}^{\mathbf{0}}$ & 17 & 15 & 10 \\
\hline
\end{tabular}

\begin{tabular}{|c|c|c|c|}
\hline \multicolumn{4}{|c|}{ Día 13} \\
\hline $\mathrm{Bx}^{0} / \mathrm{T}^{0}$ & $15^{\circ} \mathrm{C}$ & $20^{\circ} \mathrm{C}$ & $25^{\circ} \mathrm{C}$ \\
\hline $15 \mathrm{Bx}^{0}$ & 3 & 3 & 3 \\
\hline $20 \mathrm{Bx}^{\circ}$ & 9 & 7 & 5 \\
\hline $25 \mathrm{Bx}^{0}$ & 16 & 10 & 10 \\
\hline
\end{tabular}

\begin{tabular}{|c|c|c|c|}
\hline \multicolumn{4}{|c|}{ Día 14} \\
\hline $\mathrm{Bx}^{0} / \mathrm{T}^{\mathrm{o}}$ & $15^{\circ} \mathrm{C}$ & $20^{\circ} \mathrm{C}$ & $25^{\circ} \mathrm{C}$ \\
\hline $15 \mathrm{Bx}^{\circ}$ & 3 & 3 & 3 \\
\hline $20 \mathrm{Bx}^{\circ}$ & 8 & 5 & 5 \\
\hline $25 \mathrm{Bx}^{\circ}$ & 15 & 10 & 10 \\
\hline
\end{tabular}

\begin{tabular}{|l|c|c|c|}
\hline \multicolumn{5}{|c|}{ Día 15} \\
\hline $\mathrm{Bx}^{\circ} / \mathrm{T}^{\mathrm{O}}$ & $15^{\circ} \mathrm{C}$ & $20^{\circ} \mathrm{C}$ & $25^{\circ} \mathrm{C}$ \\
\hline $15 \mathrm{Bx}^{\mathrm{O}}$ & 3 & 3 & 3 \\
\hline $20 \mathrm{Bx}^{\circ}$ & 7 & 5 & 5 \\
\hline $25 \mathrm{Bx}^{\circ}$ & 13 & 10 & 10 \\
\hline
\end{tabular}


FORMACIÓN DE ALCOHOL EN QUINCE DÍAS DE FERMENTACIÓN

\begin{tabular}{|c|c|c|c|c|c|c|c|c|c|c|c|c|c|c|c|}
\hline & 1 & 2 & 3 & 4 & 5 & 6 & 7 & 8 & 9 & 10 & 11 & 12 & 13 & 14 & 15 \\
\hline 15 & 0 & 1 & 1 & 2 & 3 & 4 & 5 & 6 & 9 & 10 & 12 & 12 & 12 & 12 & 12 \\
\hline 20 & 0 & 3 & 5 & 6 & 6 & 6 & 6 & 6 & 6 & 8 & 9 & 10 & 11 & 12 & 13 \\
\hline \multirow[t]{2}{*}{25} & 0 & 1 & 1 & 1 & 2 & 2 & 3 & 3 & 3 & 5 & 6 & 8 & 9 & 10 & 12 \\
\hline & 1 & 2 & 3 & 4 & 5 & 6 & 7 & 8 & 9 & 10 & 11 & 12 & 13 & 14 & 15 \\
\hline & & & & & 0.02 & 0.70 & 1.37 & 2.04 & 4.06 & 4.74 & 6.08 & 6.08 & 6.08 & 6.08 & 6.08 \\
\hline \multirow[t]{2}{*}{15} & 0 & 0 & 0 & 0 & 78 & 1 & 42 & 74 & 7 & 02 & 66 & 66 & 66 & 66 & 66 \\
\hline & & 0.02 & 1.37 & 2.04 & 2.04 & 2.04 & 2.04 & 2.04 & 2.04 & 3.39 & 4.06 & 4.74 & 5.41 & 6.08 & 6.75 \\
\hline 20 & 0 & 78 & 42 & 74 & 74 & 74 & 74 & 74 & 74 & 38 & 7 & 02 & 34 & 66 & 98 \\
\hline \multirow{3}{*}{25} & & & & & & & 0.02 & 0.02 & 0.02 & 1.37 & 2.04 & 3.39 & 4.06 & 4.74 & 6.08 \\
\hline & 0 & 0 & 0 & 0 & 0 & 0 & 78 & 78 & 78 & 42 & 74 & 38 & 7 & 02 & 66 \\
\hline & 1 & 2 & 3 & 4 & 5 & 6 & 7 & 8 & 9 & 10 & 11 & 12 & 13 & 14 & 15 \\
\hline 15 & 0 & 1 & 1 & 2 & 4 & 5 & 7 & 9 & 10 & 12 & 12 & 12 & 12 & 12 & 12 \\
\hline 20 & 0 & 0 & 0 & 0 & 2 & 3 & 4 & 5 & 6 & 8 & 10 & 11 & 13 & 15 & 15 \\
\hline \multirow[t]{2}{*}{25} & 0 & 1 & 1 & 1 & 2 & 2 & 3 & 3 & 4 & 6 & 8 & 10 & 15 & 15 & 15 \\
\hline & 1 & 2 & 3 & 4 & 5 & 6 & 7 & 8 & 9 & 10 & 11 & 12 & 13 & 14 & 15 \\
\hline \multirow{3}{*}{15} & & & & & 0.70 & 1.37 & 2.72 & 4.06 & 4.74 & 6.08 & 6.08 & 6.08 & 6.08 & 6.08 & 6.08 \\
\hline & 0 & 0 & 0 & 0 & 1 & 42 & 06 & 7 & 02 & 66 & 66 & 66 & 66 & 66 & 66 \\
\hline & & & & & & 0.02 & 0.70 & 1.37 & 2.04 & 3.39 & 4.74 & 5.41 & 6.75 & 8.10 & 8.10 \\
\hline 20 & 0 & 0 & 0 & 0 & 0 & 78 & 1 & 42 & 74 & 38 & 02 & 34 & 98 & 62 & 62 \\
\hline \multirow{3}{*}{25} & & & & & & & 0.02 & 0.02 & 0.70 & 2.04 & 3.39 & 4.74 & 8.10 & 8.10 & 8.10 \\
\hline & 0 & 0 & 0 & 0 & 0 & 0 & 78 & 78 & 1 & 74 & 38 & 02 & 62 & 62 & 62 \\
\hline & 1 & 2 & 3 & 4 & 5 & 6 & 7 & 8 & 9 & 10 & 11 & 12 & 13 & 14 & 15 \\
\hline 15 & 0 & 3 & 5 & 6 & 8 & 9 & 9 & 10 & 10 & 12 & 12 & 12 & 12 & 12 & 12 \\
\hline 20 & 0 & 2 & 4 & 7 & 10 & 12 & 12 & 13 & 13 & 15 & 15 & 15 & 15 & 15 & 15 \\
\hline \multirow[t]{2}{*}{25} & 0 & 1 & 3 & 4 & 5 & 9 & 11 & 12 & 12 & 13 & 15 & 15 & 15 & 15 & 15 \\
\hline & 1 & 2 & 3 & 4 & 5 & 6 & 7 & 8 & 9 & 10 & 11 & 12 & 13 & 14 & 15 \\
\hline \multirow{3}{*}{15} & & 0.02 & 1.37 & 2.04 & 3.39 & 4.06 & 4.06 & 4.74 & 4.74 & 6.08 & 6.08 & 6.08 & 6.08 & 6.08 & 6.08 \\
\hline & 0 & 78 & 42 & 74 & 38 & 7 & 7 & 02 & 02 & 66 & 66 & 66 & 66 & 66 & 66 \\
\hline & & & 0.70 & 2.72 & 4.74 & 6.08 & 6.08 & 6.75 & 6.75 & 8.10 & 8.10 & 8.10 & 8.10 & 8.10 & 8.10 \\
\hline \multirow[t]{2}{*}{20} & 0 & 0 & 1 & 06 & 02 & 66 & 66 & 98 & 98 & 62 & 62 & 62 & 62 & 62 & 62 \\
\hline & & & 0.02 & 0.70 & 1.37 & 4.06 & 5.41 & 6.08 & 6.08 & 6.75 & 8.10 & 8.10 & 8.10 & 8.10 & 8.10 \\
\hline 25 & 0 & 0 & 78 & 1 & 42 & 7 & 34 & 66 & 66 & 98 & 62 & 62 & 62 & 62 & 62 \\
\hline
\end{tabular}


